Journal of Social Sciences 7 (4): 666-670, 2011

ISSN 1549-3652

(C) 2011 Science Publications

\title{
Block in volleyball with the help of Bodily Communication
}

\author{
Gaetano Raiola \\ Department of Human, Philosophical and Formation Sciences, \\ Faculty of Science of Education, University of Salerno, Italy
}

\begin{abstract}
Problem Statement: In volleyball, the ball is constantly fast and often the single game action cannot be understand due to the motor response that are very quick. The focus is always the time occurs between the stimulation, including the feedbacks and the chosen skills to play well. In this case it studies the relationship between the reaction time and tactical choices of the central blockers with the help of bodily communication. The tactical choices are two: Read block or option block. It calls read block when the block volleyball player jumps to block the spike in the middle of net in the first time after the decode of the kind of the setting by the setter. It calls option block when the blocker jumps to block the spike in the middle of net in the first time before the setter touches the ball to set it likely at the central hitter in the middle of the net. Approach: The method is descriptive research throughout performance analysis model. It utilizes the descriptive statistical model on a sample of 26 consecutive matches during a championship of the same team. Results and Conclusions: The data show the association between two tactical different choices of the jump of block role player: Option choice and read one. Both option choice and read choice are associated to the second ball going to the opposite court after the setter touches the ball in a medium range time. In conclusion it appears the possible inference and influence of the kind and time touches of the ball by the setter and so the effects of bodily communication by read choice and option one.
\end{abstract}

Key words: Time decision, tactical choice, skills, nonverbal language, setter, hitter

\section{INTRODUCTION}

Volleyball is a sport where the use of body language is very frequent, it is a sport with very narrow game space (81 square meters per team) and actions extremely rapid and dynamic, whose technical characteristics, tactics and logistics constantly implies non-verbal communication among the players of the same team and of the opposite team. The players, through perceptive and intuitive processing, decode the signs (Argyle, 1988), gestures and facial expressions of team-mates and/or of opponents in a few thousandths of a millisecond through the anticipation and interpretation of the consequences of the actions (Hall, 1966), ours own or others, confirming the proactive role of the brain that is not restricted to acquire all sensory events of the world, while it interrogates the world according to its conditions in order to predict and anticipate behavior, adjusting the sensitivity, combining messages, pre-specific and values estimated, according to an internal simulation of the expected consequences of the action (Berthoz, 2000). In particular, the decoding of signs or of facial expressions is "functional" when it refers to the same team and when communication among players is involved or that between the trainer and the athletes on the tactical intentions, strategies and the problems of the game. Decoding can be "diagnostic" when it is possible to recognize the diverse forms of non-verbal communication of the opposing team, through signs and elements that characterize the communication styles (Argyle, 1988; Hall, 1966) of athletes and coach. The third form of decoding is "tactics" when the gesture or the action simulates a game intention to solicit a reaction of the adversary who helps his own team because, in the process of observation, the sensory information is encoded as motor acts and our motor system is activated as if we carry out actions that we observe, involving the simulation of the same and making possible that 'reciprocity' of acts and intentions which is basic for the immediate recognition by us of the meaning of the gesture of others "as soon as" we see someone to perform an act or a chain of acts, its movements, which it wants to or not, it gets an immediate meaning for the observer. The system of mirror neurons and the selectivity of their answers determines a shared action space (Iacoboni, 2008), where every act and every chain of acts, our's own or others, are immediately written and understood, without any express or deliberate learning 
process" (Rizzolatti and Sinigaglia, 2006). Bodily communication can be helped by performance analysis method and technological system.

Performance analysis for very complex performances in technical disciplines is complicated. The first analysts have traditionally focused on team and match-play sports and various techniques have been effectively used to collect and analyze data in these sports (Hughes and Bartlett, 2002). However, there is no evidence in the literature of computerized real-time analysis used in rhythmic gymnastics and other type of sports with movement composition. Second, the time cost to obtain the necessary quantitative data from video digitization is so high that the advantages of immediate feedback are not available to the athlete and to the coach.To facilitate the immediate feedback benefits to improve performance, rapid performance analysis methods must be developed. Therefore, the Performance analysis could help to evaluate with a new way the effects of bodily communication in sport so fast as volleyball. To investigate on the relationship between the reaction time and tactical choices of the blockers with the help of bodily communication. The tactical choices are two: Read block or option block. Read block when the block volleyball player jumps to block, after the decode of setter's set, option block when he jumps to block before the setter touches the ball to set likely at the hitter in the middle of the net.

In volleyball the ball is constantly rejected by the players in little court and the speed of it is too high to be well perceived by athletes always in different context. The players often unconsciously reject the ball and then they exactly understand how they shot. Sometimes the insights of the player are helped by the encoding and decoding process. In these cases, bodily communication message can help the players to anticipate the exact data of decoding and then to give an immediate shot. Motor control research has not investigated yet on which role the bodily communication has, included the feint, on the rejecting a ball in volleyball and the effects of it on the final skill and its influence on outcomes. Particularly, bodily communication could be the players that anticipate the decision to give a shot and reduce the reaction time of the blocker in opposing to the attacker. The blocker has normally two choices for constraining the attacker: The first, anticipating the jump without to analyze the data and it is called option block and the second, to jump after to analyze data and it is called read block. The aim of this study is to investigate what choice the blocker plays for every skills and what is the association between tactical choice and reaction time. We analyze three volleyball skills: The off speed hit goes over the opposing block, the second ball to the opposite court and the powerful spike as fast as possible in the middle of the net.

\section{MATERIALS AND METHODS}

Descriptive research throughout performance analysis model by a complex model. Hand notation in real time and video software notation in deferred time. Statistical model on a sample of 26 matches during a championship of the same team. By video analysis with slow down mode and go back mode, it analyzes the reaction time of the blocker at the three volleyball skills: (a) the second ball to the opposite court (Fig. 1), (b) the off speed hit goes over the opposing block (Fig. 2),

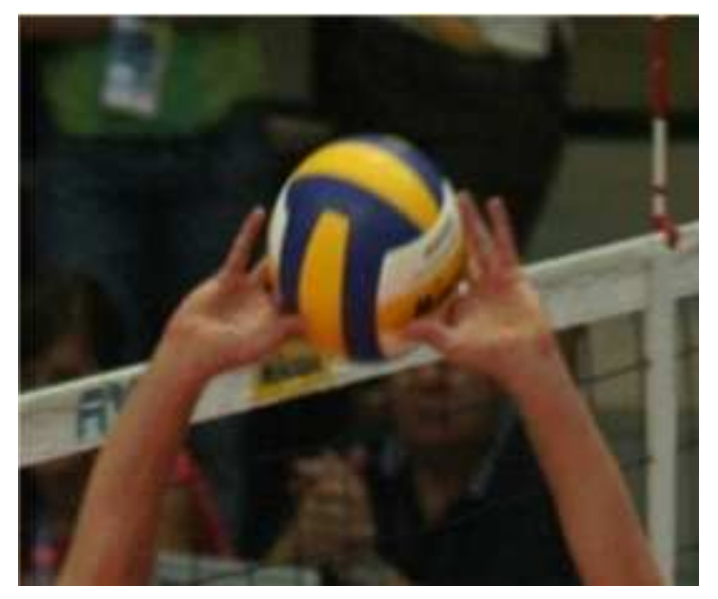

Fig. 1: The second ball to the opposite court with c) after the setter touches the ball

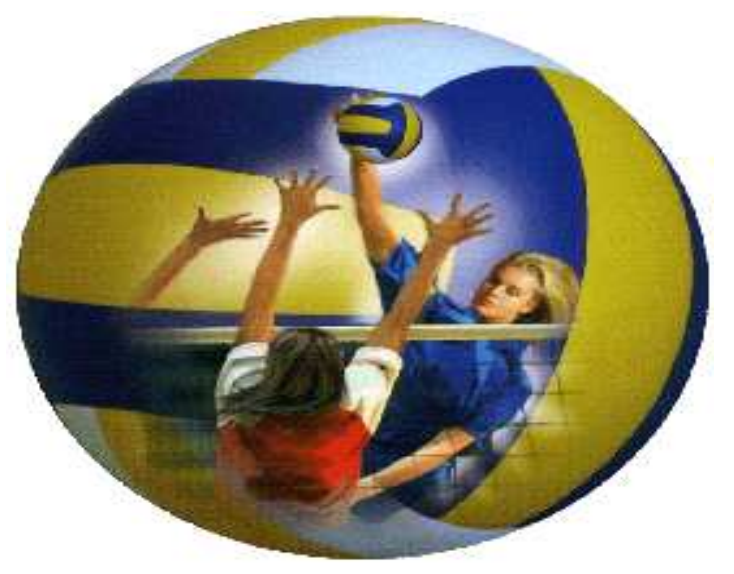

Fig. 2: The off speed hit goes over the opposing block with b) when the setter touches the ball 


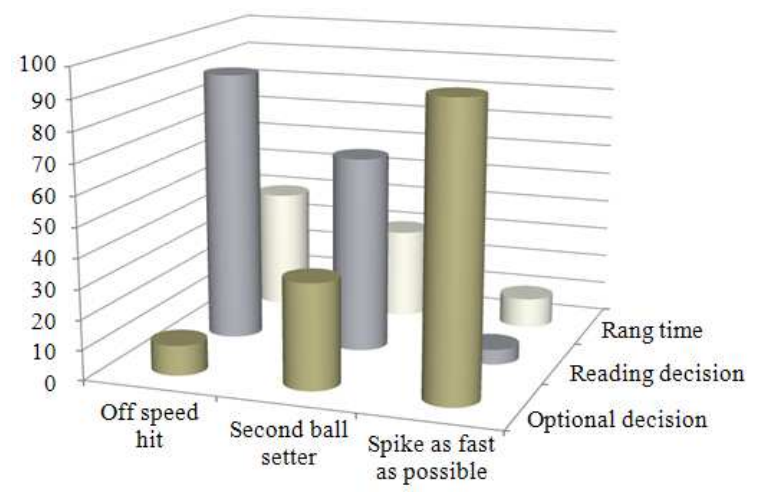

Fig. 3: Association among tactical choice-three bodily communication skills-range time

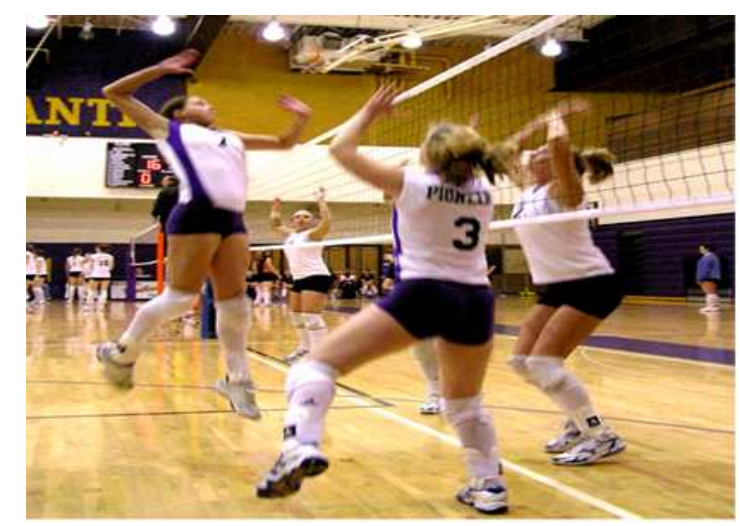

Fig. 4: The powerful spike as fast as possible in the middle of the net with (a) before the setter touches the ball

(c) the powerful spike as fast as possible in the middle of the net (Fig. 4). The reaction time is ranged in three kinds: (a) before the setter touches the ball, (b) when the setter touches the ball and (c) after the setter touches the ball. Finally, to associate the tactical choices of the blocker of option and read one at the kinds of the reaction time and the single skill. By video analysis volleyball matches with slow down and go back mode, we estimate the tactical choice of the blocker relating at the different three skills. Furthermore, we calculate the time that is between the end of one of each skill and the start. We divide the time in three ranges: Before the shot (A), almost in same time to the shot (B) and after the shot $(\mathrm{C})$. Finally, to associate the reaction times for constraining to each skill and the tactical choice.

\section{RESULTS}

Firstly, the data show the relationship between anticipating start of the blocker jump and option choice for (3) the powerful spike as fast as possible in the middle of the net with (a) before the setter touches the ball. Secondly, they show the relationship between the post blocker jump and read choice for (2) the second ball to the opposite court with c) after the setter touches the ball. Finally, there is the ambiguous relationship in a read and option choice for (1) the off speed hit going over the opposite block with (b) when the setter touches the ball. It shows that the block response is in $90 \%$ read block and $10 \%$ option block relating the off speed hit and reaction time is $\mathrm{B}$, it is $65 \%$ read and $35 \%$ option relating the second ball and reaction-time is $\mathrm{C}$, it is 5\% read and $95 \%$ option relating the powerful spike as fast as possible and reaction time is A. Therefore, there is significance between reaction time and tactical choice. The data show the association between two tactical different choices of the jump of blocker role player: Option one and read one. Option choice is associated to the powerful spike as fast as possible in the middle of the net before the setter touches the ball with a short range time. Read choice is associated to the off speed hit goes over the opposing block with a long range time. Both option choice and read choice (but different percentage) are associated to the second ball goes to the opposite court after the setter touches the ball with a medium range time (Fig. 3).

\section{DISCUSSION}

Usually, the phases of game that differentiate the successful from the unsuccessful teams are determined according to the analysis of the situation parameters of matches in team sports (Eom and Schuttz, 1992). For that purpose, matches or sets are used as entities inside such as the quality of performances of recorded phases or volleyball elements is analyzed. Bodily communication could determine the outcomes for the actions and skills that we have just been examined. Furthermore, in volleyball there are six players in one field that, depending on their playing role, are placed in six different zones and they are rotating clockwise during a game always for one zone, that is, it happens for every step of rotation. For this reason, the volleyball is very variable. In order for a team to be successful in the end, it is necessary to achieve the balance of performance in every actions and for every player. The data obtained with the analysis of the situation efficiency of every phase of a game, included those that we have just analyzed, are used as main guidelines in the planning and programming of volleyball training. The analysis of situation efficiency in single actions and skills and other explains of the reasons of success. 


\section{J. Social Sci., 7 (4): 666-670, 2011}

Both systems help to get in real time or after short time detailed information about important performance parameters for the skills that we have just wrote. So, the systems can help to make the trainings process more effective.

Further research and software development will focus on quantifying the critical performance values and expanding the software for other actions and skills.

The aspects of non-verbal communication in volleyball, its codes and the technical and tactical usage of gesture, are the result of the meeting between tacit and implied dimension that comes from experience gained on the range of activities and a theoretical dimension that scientifically characterizes and justifies the expressive and communicative potential of body. This study helps:

- To define a first inventory of signs used more frequently in volleyball according to the principles of bodily communication (Argyle, 1988), the aspects of perceptive senses (Berthoz, 2000) and the neurobiological implications (Rizzolatti and Sinigaglia, 2006)

- To give a general plan for training that includes knowledge about the function of gestures, signs and mimicking, the structure and the mean of nonverbal messages and the effects of bodily communication on behavior in order to build expressive and communicative skills of volleyball coaches. The paths of training for team sports coaches reflect the guidelines of studies and research on the methodology of the training of volleyball focused on the improvement of functional aspects, on the development of conditional abilities and energy mechanisms and on optimization of the bio-mechanical aspects, undervaluing the function of bodily communication during the game through the gestures, the signs and the mimicking

This trend produces a basic formation of sports operators providing skills on the theory of training, tactics and schemes of games, the knowledge of biomechanics and automatic technical-sport gestures, functional mechanisms to recruit energy, psychological bases that regulate the action team during phases of game, but it doesn't deal with the function of the signs and the gesticulation in communication processes and in particular the "pragmatic" side of communication and the effects that it has on behavior (Watzlawick et al., 1967).

It appears the possible inference and influence of the kind and time touches of the ball by the setter and so the effects of bodily communication by read choice and option one. The association between the start of the blocker jump and the tactical choice could be determined by the time that the setter touches the ball and by the bodily communication of the setter and the attacker. The anticipating decoding of the hitter shot and of the setting of the setter is good to give a motor skill response for the outcomes. The skills in volleyball are in strict dependency of nonverbal language and it is useful replay the investigation in addition to interview the player about it.

The association of reaction time data and tactical choice suggests a particular method of training to expect the shot and make planning of training with a multiple steps on bodily communication. Therefore, it is necessary to provide an educational course for technicians and coaches that teaches and speaks about the following subjects:

- Neurobiological knowledge on the mechanisms of regulation of the various types and ways of imitation, learning and gestural communication according to the research on mirror neurons (Rizzolatti and Sinigaglia, 2006)

- Knowledge of the mechanisms of perception and on the sense of movement or kinaesthesia which makes possible of simulations and anticipations of motor actions (Berthoz, 2000)

- Knowledge of the mechanisms of imitation in the different phases of training

- Acquaintance and meaning of non-verbal communication, of the interpersonal motor attitudes, of posture, of facial expressions, of contact, of walk and of the glance (Argyle, 1988)

\section{CONCLUSION}

Bodily communication and Biomechanics plays an important role in optimizing the training quality in elite sport. The areas of applying biomechanics in the training process of elite athletes are very diverse. In the first area those parameters which essentially influence performance have to be analyzed. These investigations have to be done during field studies using highly developed kinematic, kinetic measurement systems. The scientific challenges lie in the precision of the parameters determined (accuracy of the measurement system) and in the fact that the athlete should not be interfered severely by the measurement system during performance. The results of such investigations lead to a better understanding of movements used and provide coaches and athletes valuable support in directing training goals. 


\section{REFERENCES}

Argyle, M., 1988. Bodily Communication. 2nd Edn., Taylor and Francis, Madison, ISBN: 0416381502, pp: 363

Hall, E.T., 1966. The Hidden Dimension. 1st Edn., Garden Peter Smith Pub, Gloucester, ISBN: 0844665525, pp: 217.

Berthoz, A., 2000. The Brain's Sense of Movement. 1st Edn., Harvard University Press, Cambridge, ISBN: 0674009800, pp: 337.

Iacoboni, M., 2008. Mirroring People. The New Science of How We Connect With Others. 1st Edn., Farrar, Straus and Giroux, New York, ISBN: 0374210179, pp: 308.
Rizzolatti, G. and C. Sinigaglia, 2006. So Quel che Fai. Il Cervello che Agisce e i Neuroni Specchio. 1st Edn., R. Cortina, Milano, ISBN: 8860300029, pp: 216.

Hughes, M.D. and R.M. Bartlett, 2002. The use of performance indicators in performance analysis. J. Sports Sci., 20: 739-754. PMID: 12363292

Eom, H.J. and R.W. Schuttz, 1992. Statistical analyses of volleyball team performance. Res. Q. Exerc. Sport, 63: 11-18. PMID: 1574656

Watzlawick, P., J.B. Beavin and D.D. Jackson, 1967. Pragmatics of Human Communication: A Study of Interactional Patterns, Pathologies and Paradoxes. 1st Edn., W W Norton and Co Inc., New York, ISBN: 0393707075, pp: 304. 